\title{
Case Report: Implications of Doing Research on Socially Assistive Robots in Real Homes
}

\author{
Susanne Frennert $^{1} \cdot$ Håkan Eftring $^{1} \cdot$ Britt Östlund $^{2}$
}

Accepted: 11 January 2017 / Published online: 3 February 2017

(C) The Author(s) 2017. This article is published with open access at Springerlink.com

\begin{abstract}
The current paper addresses the implications of doing research on socially assistive robots in real homes. In contrast to laboratory studies, studies of robots in their intended natural environments can provide insights into people's experiences of robots, and if and how a robot becomes embedded and used in people's everyday life. However, moving robots out of the lab and into real life environments poses several challenges. Laboratory methods mainly focus on cause-and-effect relations between independent and dependent variables, while researchers who are conducting studies in real homes have much less control. In home trials, researchers need to decide what kind of data is obtainable and available. In real homes, researchers face unique challenges that require unique and pragmatic approaches. Any single study conducted in a real home is likely to have methodological limitations. Therefore, several different studies using different robots and methods are needed before the results can be converged in order to reach conclusions that are convincingly supported. This paper is an effort to provide such a report on a specific empirical case and converging findings from other studies. The goal is to provide an account of the research challenges and opportunities encountered when introducing a robot into its intended practice: the homes of older people. The aim is to give enough details for other
\end{abstract}

Susanne Frennert

Susanne.frennert@certec.lth.se

Håkan Eftring

hakan.eftring@certec.lth.se

Britt Östlund

britt.ostlund@kth.se

1 Department of Design Science, CERTEC, Lund, Sweden

2 School of Technology and Health, KTH Royal Institute of Technology, Stockholm, Sweden researchers to critically examine and systematically build on the insights and findings presented.

Keywords Social robots - Older people Science and technology studies · Case report - Everyday life

\section{Introduction}

There is a longstanding interest in understanding how robots can entertain, cure, care for and/or support older people at home. The implementation of robotic solutions can offer opportunities to enhance the quality of life and improve the efficiency of care at home, but it also raises questions and doubts. New areas of concern and obstacles surface as new robotic solutions are developed and deployed. Sharkey and Sharkey [59] warn that robots in eldercare will reduce time for human contact, deceive older people into believing that they are being cared for, increase the feeling of loss of control, privacy and personal liberty. On the other hand, others argue that robots can provide 24-h support and assistance and that an increased ageing population means that society will no longer be able to provide human care for all older people in need [37]. The increased digitalisation in society has resulted in social changes in which human experiences are progressively mediated by technology. As a result, digitalisation is altering human communication, actions and practices. Research in the field of human-computer interaction (HCI) shows that well-designed technology can enhance people's everyday life, while badly designed technology comes with well-known risk factors that can lead to stress, frustration and anxiety [18]. An understanding of the effects of a specific technology can only be achieved by implementing the technology in its natural environments, which is often referred to as "studies in the wild" $[11,13,56]$. The problem with devel- 
oping useful technologies is not the technology per se, but an understanding and knowledge of how potential users make sense of the technology [63].

In the field of human-robot interaction (HRI), robots are mostly studied in lab environments with students as participants [4]. The results from such studies are questionable since they may not be applicable in natural environments. In parallel, it is also questionable whether robots are ready to move out of the lab and into natural environments, due to technical problems that have yet to be resolved, in order for autonomous socially assistive robots to act and interact with people in real life [4].

User acceptance, revelations and experiences are important means for socially assistive robots to gain market share and widespread adoption. While robots with limited sets of functionalities have been tested in real life environments $[24,65]$, more advanced robots have only been tested in natural environments for a few limited hours a day or with restricted autonomy [21,52]. Several researchers have highlighted the need for long-term studies of robots in natural environments $[7,9,26,62]$. It has also been argued that the development of robots for older people needs to involve them in the design process [26]. One way to do so is by letting them try out a robot in their own homes in order to explore how they perceive the robot and if it becomes integrated into their routines and habits of everyday life. These kinds of studies entail methodological challenges on how to collect the data in the participants' homes. Questionnaires, interviews, sensor and robot logs are usually used [57]. Sensor and data logs are perceived as objective ways of collecting data, since they do not involve researchers invading the homes of the participants. However, they do not provide information about a participant's motivation for participating. Nor do data logs reveal why participants use certain functionalities and not others.

There is a gap in the field of HRI in the understanding of how older people use and integrate a multifunctional socially assistive robot into their everyday life and what factors need to be considered when conducting home trials. The case study presented in this paper addresses the gap by placing a socially assistive robot prototype in seven older women's homes and letting them explore it freely without any guidance or restrictions. They tested the robot for three consecutive weeks. Our analysis of the results makes two primary contributions to the field of HRI: (1) Studying older people's day-to-day experiences of having a robot at home provides a lens through which one can explore how older people engage with robots. From this we argue for a focus on incremental robotic development during home trials: (a) because the participants' experiences, needs and wants develop and evolve during the trial period, and (b) because there is no point in continuing long-term trials without correcting technical malfunctions since these will affect the participants' usage of the robot, their experience and the role they ascribe the robot. (2) We contrast our findings with the existing literature on socially assistive robots and home trials, converging findings from other studies.

\section{Relevant Work}

Numerous robots to prolong independent living have been developed and studied as part of the trend of ageing at home [5]. Examples include the EU project "CompanionAble" in which a companion robot was developed for older people with mild cognitive impairments [58]; the ALIAS robot to increase social inclusion [52]; and a cognitive care robot for elderly assistance [21]. Both field studies and laboratory studies have demonstrated that robots can stimulate significant emotional attachment, which is further strengthened if the robot says the person's name or positively responds to the person's interaction with it $[20,32,67]$. In addition, studies with PARO (an robotic baby harp seal), which is used as a therapeutic device, indicate that interaction with the robotic seal has positive effects on quality of life, feelings of pleasure, and contributes to overall well-being $[6,10]$. Other results show that the participants feel less lonely and depressed if $P A R O$ is used at the care facility where they reside $[30,53]$. Robots can efficiently be used as motivational coaches, such as helping people to stay on a diet [32].

Research shows the importance of assessing the expectations and needs of a range of stakeholders (older people, family, medical staff) [7]. This is further confirmed in a literature review by Bemelmans et al. [6] who also claim the need for all the stakeholders to understand the benefits of the robot in order for it to be accepted. Kidd et al. state that a supporting socio-material setting is important for the acceptance of a robot in a healthcare context [31]. Heerink et al. [28] present a model on how to assess older adults' acceptance of assistive social technology. Their model has been tested in several studies with older people. They propose that the use of robots - what they refer to as "assistive social agents" - is determined by social influences, facilitating conditions, perceived usefulness, perceived ease of use, perceived enjoyment, attitude and trust. Likewise, Young et al. [72] confirm that acceptance of robots is dependent on subjective user perceptions of what robots are, how they work and what they can and cannot do. They concluded that the factors affecting acceptance are: safety, accessibility, usability, practical benefits, fun, social pressure, status gains, and the social intelligence of the robot.

Most of the research in the field of HRI in terms of preferences and attitudes about robots has been based on questionnaires without the potential older users having experience of robots [62]. Home trials with commercial robots (e.g. vacuum cleaning robots) have added valuable findings and have shown that robots do not exist in isolation, but in 
Fig. 1 The HOBBIT prototype
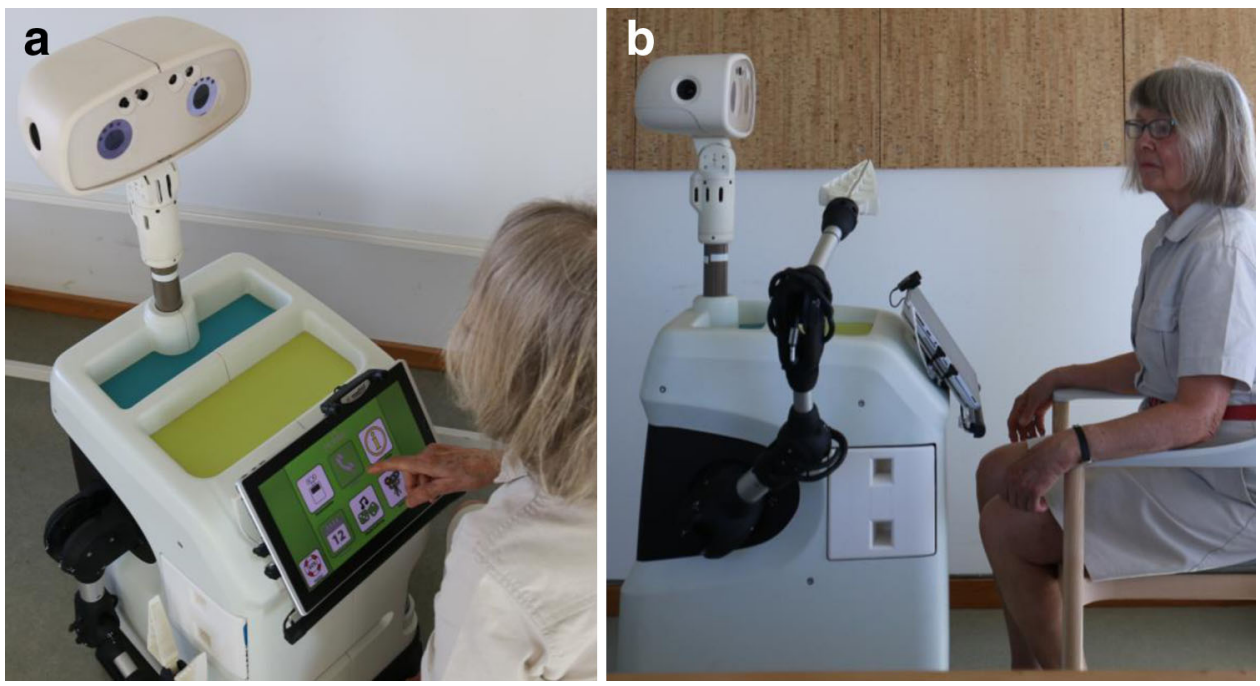

relation to their use context and to a broader cultural context $[24,64]$. While home trials with robots with a limited set of functions can add to our understanding of the acceptance and usage of a specific function and the ecological factors that impact this acceptance, more studies in natural environments with multifunctional robots are needed [62]. In eldercare, robots are foreseen as being able to augment either older people's physical (physical assistance) or cognitive abilities (reminders, companions, etc.) or both.

The robot prototype used in the case study presented aims to provide both physical and cognitive assistance for older people in their own homes. Testing a multifunctional robot in real homes involves many challenges and considerations. To our knowledge, this study is the first of its kind. We will present insights into the internal and external factors that can complicate the interpretation of data collected during home trials of a multifunctional robot.

\section{The Case Study}

A case study was carried out between May and August of 2015. The methods used were participatory observations, participant diaries, semi-structured interviews and questionnaires to explore the day-to-day experiences of seven older people of an assistive service robot in their homes. The fieldwork was carried out in a EU funded project called HOBBIT. ${ }^{1}$ Home trials of a total of 18 users were carried out in Sweden, Austria and Greece. The trials in all three countries involved questionnaires and interviews, while in Sweden we added observations and diaries. The project aimed to develop a robotic system that assists and enables older people to live in their homes for a longer period. The robot prototype had a

\footnotetext{
${ }^{1}$ www.hobbit-project.eu.
}

mobile platform, multiple sensors, an arm with a gripper and a multimodal user interface (Fig. 1). The robot also had features such as human detection, tracking, gesture recognition, gripping and learning. It was developed to prevent falls and carry out the following tasks: pick up objects on the floor; transport objects from room to room; remind the user to take medication, drink water, and attend important meetings, etc.; clear the floor; find and retrieve objects; show physical exercises; detect falls by patrolling; and make emergency calls.

The robot had pre-programmed locations that it went to when the user pressed the call buttons, or the go-to locations on the touch screen, or called (voice control) for the robot to come. The robot had a calendar where the user enter appointments, or reminders about when to drink or take medications. Thirty minutes before a set appointment the robot located the user (by autonomously moving to preprogrammed patrolling areas) and told her about the calendar event. The robot also provided entertainment such as access to the Internet, local news, eBooks, music and games. The robot patrolled the user's home every third hour to check if she was all right. It moved autonomously from one patrolling area to another and asked the user if she was there. If the user did not respond, the robot called pre-programmed numbers for relatives or neighbours to let them know that it could not find the user. For more details regarding the project, the robot prototype and results of the questionnaires and quantitative studies see $[1,3,19,23,36,39,40,47,50]$. In this paper, we will provide insights from the home trials carried out in Sweden.

\subsection{Participant Selection and Description}

The older participants were recruited through advertisements and word of mouth. As such, they were a self-selected group who were interested in testing a robot at home. The inclu- 
Table 1 The participants

\begin{tabular}{|c|c|}
\hline Participant & Description \\
\hline Nr. 1 & $\begin{array}{l}\text { A } 90 \text {-year-old woman living on her own. Before retirement she worked as a physical education teacher. } \\
\text { She practiced swimming, yoga and line dance every week. She uses her computer daily and is very } \\
\text { interested to in learning about new technologies }\end{array}$ \\
\hline Nr. 2 & $\begin{array}{l}\text { A } 78 \text {-year-old woman living on her own. Before retirement she worked as a teacher. She is interested in } \\
\text { changes in society and in technology. She frequently uses her computer }\end{array}$ \\
\hline Nr. 3 & $\begin{array}{l}\text { A } 78 \text {-year-old woman living on her own. Before retirement she worked as an engineer. She often looks } \\
\text { after her grandchildren. She lives in an apartment building for older people and eats her lunch in the } \\
\text { resident dining room. She uses her smartphone and computer every day }\end{array}$ \\
\hline Nr. 4 & $\begin{array}{l}\text { A 76-year-old woman living on her own. Before retirement she worked as an artist and as an assistant at a } \\
\text { school. She uses her smartphone and tablet every day }\end{array}$ \\
\hline Nr. 5 & $\begin{array}{l}\text { A } 78 \text {-year-old woman living on her own. Before retirement she worked at the city library. She often looks } \\
\text { after her grandchildren and frequently uses her computer }\end{array}$ \\
\hline Nr. 6 & $\begin{array}{l}\text { An } 82 \text {-year-old woman living on her own. She worked at a bank before retirement. She uses her computer } \\
\text { frequently and helps other people with their finances }\end{array}$ \\
\hline Nr. 7 & $\begin{array}{l}\text { A } 85 \text {-year-old woman living on her own. She has lunch every day at the restaurant on the ground floor. } \\
\text { She has severe knee problems, which makes it impossible to walk } 500 \mathrm{~m} \text {; she also has severe sight and } \\
\text { hearing problems. She does not own a computer and has never used one }\end{array}$ \\
\hline
\end{tabular}

sion criteria for selecting participants out of the group of 28 people who responded, were as follows: (1) living on their own, (2) over the age of 75; and (3) had fallen sometime in the last 2 years or were afraid of falling. Given the nature and complexity of implementing and running test sites with an assistive robot in the making, the participant selection was not based on random sampling but on the older person's willingness, suitability and preparedness to allow us to use their homes as a test site. Once a potential participant was identified, he or she was given additional information about the study. We visited their homes to assess if the participant fulfilled the criteria and if the home was suitable for the trials. All the potential households were rated and the ones that best fit the inclusion criteria were selected. All participants signed a consent form and were reassured that they could change their minds at any time and the robot would be removed without question or explanation. A total of seven participants were included in the study. They were between 76 and 90 years of age. Their technology experience ranged from frequent use of tablets and smartphones to no experience of using a computer or a smartphone. All the participants were women. Women have a higher life expectancy and are less likely to remarry than men, and as a result, more older women than men tend to live alone in old age [17]. The participants are briefly described in Table 1.

\subsection{Data Collection}

The insights presented in this paper are grounded on the data collected from three data sources: (1) semi-structured interviews; (2) diaries; (3) observations. Empirical data was collected at three different stages: before the robot prototype was installed; during the time the robot prototype was installed and used at the participant's home; and after the robot prototype had been removed.

\subsubsection{Semi-structured Interviews}

Each household was visited at least four times for semistructured interviews: once before the robot was introduced, once in the middle of the trial period, once towards the end of the trial period, and once about a week after the trial ended. Demographic data was gathered in the pre-introduction interviews. On the first visit, we explained that the purpose of the project was to understand how older people use a robot in their home in terms of attitude, perceived safety, utility, flexibility, self-efficacy and emotional attachment. The interviews were modelled after ethnographic interviewing [2] and followed predetermined topics such their expectations of the robot, biography of the individual (social, cultural and historical context), experience of ageing, important people in their lives, experience of ill health and emergencies, and of other technologies. The subsequent two interviews, during the time each participant had the robot at home, were also modelled after ethnographic interviewing [2] and followed predetermined topics such as their perceived usage and usefulness of the robot, their views on how the robot and other robots could be used and could support independent living in the future, other interests and activities related to everyday practices and the robot, other technology usage and healthcare issues. The post-interview (after the robot had been removed) focused on 
the participants' experiences of partaking in the home trial and on what was important and significant for them during the trial. In preparation for each interview we reviewed our notes from prior interviews. In addition, we asked friends and relatives follow-up questions via the phone in situations in which clarifications were required. The researchers regularly summarised their interpretations of what was said during an interview to enable the participant to clarify responses and to correct misconceptions. Each interview lasted between 60 and $90 \mathrm{~min}$. The interviews were taped to which the participants had given a signed consent before the start of the trial.

\subsubsection{Diaries}

The older participants were asked to document their usage by using a camera and keeping a diary. They were requested to reflect in the diary every day on questions such as: Did you use the robot today? What functionalities did you use? Why did you use the robot? How often did you use the robot today? What was your experience of using the robot? Four of the participants filled in the diaries every few days while two only filled them in a few times. One woman did not provide a diary due to her visual impairment. The participants' diaries were discussed during the post-interview. Keeping a diary turned out to be a great means of eliciting what was important and significant for the participants about the robot. It also reminded them of specific events they had documented but had forgotten to mention in the interviews.

\subsubsection{Observations}

The researchers also observed and documented how the participants interacted with the robot during their visits. The initial observations were incorporated into working with the set-up and installation of the robot. As a result, we observed how the usage of the robot evolved over time. The participants were observed while learning about the robot, interacting with it, and using it on several occasions. Observations were conducted two to five times at each test site and lasted $120 \mathrm{~min}$ on average. The participants were observed during the course of their daily activities. The researchers sat with the participants at their dining or coffee tables, took field notes about their interactions with the robot and the functions they used, and occasionally asked questions to clarify what the older person was doing. In some cases, the participants were asked to show how they "normally interacted with the robot". They were asked to walk the researchers through their interactions with the robot and its interface in order to demonstrate the functionalities, how they responded to the robot's feedback, and how they used the robot. On other occasions the partic- ipants were asked to go about their day as if we were not there.

\section{Findings}

This section presents the information generated on the internal and external factors that complicate the interpretation of data collected during the home trials. Both factors are related to the structure and design of the research project, and not to the individual participants.

All the interviews were recorded and transcribed. Field notes were taken during the direct and participatory observations and during all other home visits. These included memos and reflections. The qualitative data analysis software, NVivo, was used to organise all the collected data including the participants' diaries, interview transcripts and observational field notes. Photos, digital maps and audio files were also collected and stored. All the texts were subjected to qualitative content analysis [27]. The units of analysis were based on interview texts, observational field notes, and diary texts. Due to the extensive amount of material, summaries were written very close to the data in which long relevant quotes were included. Relevant data was grouped together in a grid in order to identify patterns (to identify the individual participant's consistency of message) and contradictions for each participant and between the different data sources (interviews, diaries and observations). The interviews and observational field notes were the main tools, while the diaries were used as a supplement to validate the findings.

Cross-examination of the material began after all analyses of each participant were completed. Similarities and differences were identified. Emerging relationships were redefined through replication logic, revisiting the data to see if each participant demonstrated the same pattern. The analysis process was iterative. Charts and tables were used to facilitate comparisons between the different participants and data sources. The analysis revealed several interesting insights and design challenges that can be beneficial to understand when designing and evaluating future socially assistive robots. The richness of the data means that it is relevant to a number of discussions. This paper will discuss only a subset of the results: the relevant aspects to be reflected on when conducting home trials and analysing the results. The internal factors are described first and then the external factors.

\subsection{Internal Factors}

Internal factors are the aspects that researchers in a research project can influence and change. The main internal factors identified are: the design of the robot, feedback and visual clues, the fit between the robot and the home, and the research design. 


\subsubsection{The Design of the Robot}

Robots exist in all kinds of shapes, sizes and with all kinds of functionalities $[15,59,68]$. Depending on the design and behaviour of a robot, people will react differently to it. People's acceptance and preferences of one robot are not easily generalised or compared to another robot with a different behaviour and/or design. There was a homogenous response to the robot in our study. All of the participants attributed human traits, emotions and intentions to it. For many, this was due to its design (i.e. eyes, head, arm). A statement of participant 5 captures the influences the design had on her perception of the robot:

$\mathrm{He}$ is very cute. The eyes are probably very important. The eyes make me feel empathy and he makes me laugh. Then he moves and his head moves. It makes me happy and compassionate. I think that I want to see him as something that is alive. He's charming.

Here participant 5 describes her desire to perceive the robot as something real and alive. Similar anthropomorphism has been observed in previous human-robot interaction studies $[20,32,67]$, and in human-computer interaction and media studies [51]. Fear has been raised that robots can deceive older people into believing that robots generally love and care for them [61]. However, in our study it was obvious that all of the participants considered the robot a machine and not a living creature, but attributed human traits to the robot while talking about it. For some, anthropomorphism was related to the robot's behaviour (i.e. perceived as stubborn, kind or having a will of its own), while for others it was related to the robot's utility of talking with a human voice. The autonomy and design of the robot evoked the participants' readiness to interact with it as with other humans. However, the robot prototype did not have any inherent social abilities or was able to react reciprocally to the users' interactions. Its behaviour, reactions and interactions were preprogramed; still all the participants initially perceived the robot as social. They all wanted to interact with the robot by talking to it. However, although all of them attributed human traits to the robot, a gap was observed between the participants' perceptions and expectations of the robot and its actual abilities. When the participants talked to the robot, they found it difficult to hear and understand its responses. For some, the robot did not respond to their verbal commands. For others, the robot executed actions that they did not understand or that they had not asked of it. Most of the participants initially altered their behaviour to suit the robot by changing how they talked to it by trying different kinds of voices, dialects, wording and sound levels. Thus, their solution when they did not achieve the expected result was to use the touch screen.
Past research indicates that for acceptance, a robot should not be too big or bulky, should have human traits, but should not resemble humans to the point that the user expects it to behave like one [71]. It turned out during the home trials, that since the robot spoke with a human voice, the participants wanted to talk to it as with other humans; they did not want to command it, but instead to have a mutual dialogue. The robot was unable to meet the users' expectations in its ability to understand and react to human speech. Other studies have also shown that older people would like the robots they are testing to be more intelligent, social and spontaneous $[16,46,70]$. In our case study, all the participants said that they liked the appearance of the robot and that it was perceived as kind. However, the size of the robot turned out to be an issue and most would have liked it to be smaller.

\subsubsection{Feedback and Visual Clues}

One of the main promises of socially assistive robots in eldercare is to increase the social interaction and participation of older people. However, if older people do not understand the interactions and actions of the robot, this may instead cause anxiety and disbelief in their own abilities to handle robots. It may seem obvious, but an important insight drawn from the study presented in this paper is that a robot needs to be reliable and its actions need to be understood by the users when tested in real homes. Robot actions and interactions may be understood and explained from a technical viewpoint but if its users do not understand the robot's actions, a level of uncertainty and insecurity arise and affect the natural and frequent usage of the robot. This also affects the evaluation and the results [49]. Several of the participants stated that given the uncertainty and unreliability of the robot's actions and interactions, they could not seamlessly integrate it into their everyday life. For example, most of the participants stated that the patrolling and reminder functions only worked once in a while. When looking at the data logs it became apparent that this was due to the participants frequently using the "rest function" of the robot when they wanted to send it to the charging station. The rest function was originally intended to be used when the users left the home or went to sleep in order to prevent false emergency alarms. These kinds of misunderstandings are hard to detect when the participants are only trying out the robot once or in a laboratory setting with the purpose of studying human-robot interaction.

When older people have a robot at home for a longer period, they have all kinds of activities and practices that need to be incorporated with the testing of the robot, such as when they go out or take a nap and do not want to be disturbed by the robot. In other instances, they just want to send the robot to the charging station but still be reminded by the robot about 
appointments and other obligations. The robot's behaviours need to be made visual to its users to overcome these kind of misinterpretations [45]. Visual clues serve as interpreters between technical issues and the robot behaviour. The user does not need to know about the technicalities of the robot but she does need to be able to interpret and make sense of the robot's behaviours and actions. In contrast to studies in the lab where there are always people nearby, a person is alone at home and may find it stressful to try and handle a robot that behaves in a way she does not always understand. This is even more so the case if it is an autonomous robot that moves on its own every third hour in her home and which she cannot turn off.

\subsubsection{The Fit Between the Robot and the Home}

A major obstacle for the use of the robot among the participants in their own homes was the robot's lack of fit to the home environment. When developing autonomous robots for home usage, it needs to be able to move around in rooms filled with furniture, carpets, thresholds, narrow corridors, etc. All of the participants reacted negatively to the robot's movements. The robot had sensors, which served as a means to identify the surroundings and to help it navigate in the participants' homes. However, the latency between the sensor information and the robot action (to move), made the robot move incoherently and resulted in jerky movements, which negatively affected the participants' perception of its reliability. Narrow corridors and the moving or addition of new furniture during the trials resulted in the robot losing its localisation ability so that it ran into furniture and walls. When this happened it needed to be relocalised by a member of the development team. The importance of feeling safe and in control at home became apparent in these instances. All the participants wanted to be sure that they could leave their homes without the robot leaving the docking station during the time they were out. They wanted to be able to turn the robot off. But there was no turn-off function because if the system was turned off, it needed to be restarted and the cameras and arm needed to be recalibrated by a member of the development team. In carrying out home trials, many of these practical concerns became obvious, but could have easily been missed in laboratorycontrolled environments. Furthermore, in contrast to the laboratory studies [25], the participants also complained about the loud noise the robot made (from a cooling fan) in the home trials, and that it emitted light and generated heat. Other perceived barriers were that carpets needed to be removed and that the robot could not negotiate high thresholds (ramps were sometimes used causing the robot's head to move too much, in which case the head camera had to be recalibrated).

\subsubsection{The Research Design}

The research design of the home trials was decided within the project $[1,3,19,23,36,39,40,47,50]$. All home trials were to follow the same procedure in order to be able to contrast and validate the results from different sites and countries. As such, the design process followed a linear set-up in which potential users were first involved in identifying the requirements, and thereafter a first prototype was developed and tested with users in a usability lab. After that, a second prototype was developed and tested in real homes $[19,25]$. The design process differed from many others where if older people are involved, they are often given the passive role of evaluating the usability and acceptability of a predesigned robot $[43,54]$.

An important insight from our home trials is that as soon as an error or misunderstanding between the user and the robot's actions and interactions is detected and validated by other users, it should be redesigned. Long-term studies should last at least 2 months if the intention is to understand the longterm user experience and not just the initial experience and that is influenced by the novelty effect [64]. However, there is no point to continue testing a robot with the same errors for weeks or months because the participants will only report them over and over again. During our home trials it become clear that either the participants' first impressions last, or the participants become more negative during the trial period. There is also a risk that the participants will stop using the robot all together. For example, all of the participants recognised the value of the robot prototype being able to pick up objects from the floor, learn to recognise new objects and to search for missing or misplaced objects. They perceived these functions as enabling independence and autonomy. Yet, when they tested these functions, the robot's responses were often perceived as odd. Sometimes the robot could not detect an object, upon which it said that it could not even though the object was clearly visible to the participants. In other instances, the participants taught the robot to recognise an object, but when they asked the robot to find it, the object was not saved in the "objects list" (all objects that the participants taught the robot to recognise were to be saved on an accessible list on the robot). These errors were due to software problems that were not communicated to the participants and so they did not understand if the failure of the robot to carry out the tasks was due to their mismanagement or to the robot's incapability. As a result, these functions were initially tested by the participants or by first time visitors (grandchildren, friends and neighbours) but were not used on a regular basis.

The entertainment functions, which did work, were used more frequently and the robot was perceived as being more as a toy than a device that increased independent living and the feeling of safety at home. The results 
would have been different if the functions with technical problems (picking up objects, learning and detecting objects) had been improved and honed during the trial period.

\subsection{External Factors}

External factors are outside influences that impact the participants' usage and experience of a robot. They are difficult or impossible for researchers to influence and change, but still have to be considered and acknowledged when developing and evaluating a robot. The two main external factors identified in this study are competing artefacts and the motivation to participate (which includes the meaning given to the trials, to being a participant and to the robot by the participants).

\subsubsection{Competing Artefacts}

In relation to robots, independence can take on a variety of forms that include helping with day-to-day tasks such as searching for, finding and picking up objects. One of the main objectives of the robot was to prolong independent living for older people. It was thus decided from the beginning of the project that the robot should have an arm and be able to pick things up from the floor. This would be one way for the users to avoid fall accidents due to dizziness. It was also decided that the robot should be able to detect if someone had fallen. The pick-up procedure proved to be too complicated and time consuming. The following excerpt from the observational field notes describes the pick-up functionality:

It is in the afternoon. Participant 3 has had her lunch at the canteen and I arrived at her flat at around 14:30. It is a beautiful summer day and the sun is shining through the windows of her living room. The wooden floor is bare and her furniture is placed in different groups with a large space between the groups. I am sitting on the sofa trying to look occupied with my computer while I observe the participant interacting with the robot. First she presses the call button and the robot leaves the docking station. The robot says: "Coming". The participant puts her eyeglass case on the floor about 1.5 metres away from the robot. She presses the "Pick up object" icon on the touch screen and places herself 2 metres away from the robot. She stands in an upright position and points at the object. After about 30 seconds the robot says: "I have detected your point gesture and I will start searching for the object". Another 15 seconds pass and the robot says: "I have detected the object and will reposition in order to pick up the object". The robot moves to another position closer to the object. It turns its head towards the object and says: "I cannot detect an object". The participant moves the object to the place where it appears that the robot is looking but nothing happens. She waits for about 60 seconds and after that she presses the "Go to charging" icon and the robot says: "I will go to the docking station", and starts moving in that direction. It successfully finds the docking station and says: "I am charging". The participant gets her own gripper and uses it to pick up the object.

As exemplified in the excerpt, the user needed to stand in front of the robot at a distance of about $2 \mathrm{~m}$ for the sensors to detect her; she also needed to point at the object and wait for the robot to detect the object and place itself in a position to pick up it up. The object had to be of a certain size for the robot to detect it and grasp it with the gripper. The object also had to be in an empty space for the robot to place itself in the right position to grab it. The participants emphasised that they had difficulties picking up objects that were too small for them to see or that had fallen under the furniture. In such occurrences, the robot was unable to help them. Most of the participants already had a gripper they used for picking up objects from the floor. As a result, the pick-up function was not used since they already had a better alternative.

When developing robots for older people, the developer needs to understand what kind of artefacts the target groups is using at the moment. The robot has to fit into the network of other technologies and artefacts. There is no point in developing robots that have the same functionalities as the technologies or artefacts that the target group already uses. Robots need to be better or have an added advantage to the artefacts the target group already has [55]. During the trials it became apparent that participants found the robot "a bit hallow and shallow". All of the participants initially tried out the robot and its functionalities but found it difficult to comprehend its usefulness. They articulated that they liked that all the media were gathered in one place in the robot, such as the Internet, games, TV, radio and audio books, but the observations revealed that they used their ordinary TV and computer and not the robot most of the time. Similarly during the usability trials of the first robot prototype, many of the participants highlighted the benefits of having all media gathered in the robot [25]. However, at home in a familiar environment the participants easily fell back into their old media consumption habits and patterns. Our findings confirm the results of prior research $[46,70]$ showing that older people can see the potential of reminders, entertainment, telecommunication, etc., but that at the same time, they do not perceive the usefulness of a robot that provides the same functionalities that are already available in existing systems. 


\subsubsection{Motivation to Participate}

People's motivation for participating in home trials with robots can differ. Our case study shows that the participants were open to participate in the home trials because they were curious about robots and wanted to find out more. But participating was seen as a game, something fun, not because they believed that they needed a robot or wanted to change their behaviour. Instead their motivation was to represent other older people who could be in need of robots. Still, the trials turned out to be a social and valuable experience. An increased usage of the robot was noted when the participants had visitors at home. The circumstances for determining when and why a participant uses a robot need to be considered when analysing data from home trials. Below we present the meaning given to the trials, the meaning given to being a participant, and the meaning given to the robot by the participants.

A. The meaning given to the trials (not for real- just a trial)

There are difficulties in evaluating older people's natural behaviour with robots when conducting home trials because the trials are not natural situations. In acquiring new technologies in general, people often go through different stages of decision-making $[38,55,60]$. But when deciding to partake in home trials, people go through a different kind of decisionmaking process. They need to decide if they have the time and interest. In our case study the participants were informed that they could end the trial at any time without giving a reason. As such, participating in the home trial was more of an opportunity to experience a robot at home for a limited time, and not a matter of appropriating a robot that the participant had decided she needed or wanted., This is something that is important to acknowledge. Home trials provide insights into fragments of people's everyday life. The participants are given an opportunity to test a robot and in that way provide researchers and developers with greater understanding and a better chance to address their needs, wants and wishes when it comes to robots. Home trials can also support an on-going dialogue between participants and researchers if qualitative data is collected through interviews and observations.

It became obvious among the participants that participating in the trials was a social experience. The trials involved frequently meeting the research team. Many of the participants also reported increased interaction with family and friends. By participating in the home trials, the participants were listened to and became a "topic" of interest for friends and family. This strengthened their image of self. Participant 4 expressed this eloquently:

Mostly I get into a routine and become stuck in the pattern and I don't feel motivated to do new things. By having the robot at home, I started doing things that I have been planning to do but never got around to. It has been very easy to invite friends over when I have the robot here because then the focus is not on food or how my home looks, instead the robot becomes the centre of attention. The robot is the topic of discussion. I like to show off the robot. It broadens my horizons and I'm interested in knowing what others think. I'm very interested in social development and being able to influence the future.

The increased social interactions with the research team, friends and family during the home trials were described during the interviews as well as in the diaries. Partaking in the trials also affected interactions with people who the participants normally would not talk to, such as attention from the media. Having a robot at home became an opening for social interactions with others.

B. The meaning given to being a participant-ascribing different roles and purposes for participating in the trials

An interesting observation during the trials was how the participants ascribed different roles and purpose for participating. All of them saw themselves in the role of being advocates for others: older and more fragile people in need of robots. A recurring issue was how the participants cultivated the image of other old people as weaker and lonelier than themselves, and how this image was intertwined with maintaining their belief in the usefulness of future robots. As participant 2 concluded:

If I were less active and did not have many friends, then I believe that the robot could become a kind of companion. It is not a human but more like a machine friend. It provides a pastime, which is welcomed when the days feel like they will never end. It becomes more of a companion than a tablet or a phone since it is mobile and since it speaks and moves autonomously.

Insights like this about other, more fragile older people needing a robot were similar to those of previous studies $[44,70]$. The participant who had the most technical experience (a retired engineer) took it as her task to systematically evaluate the robot actions and interactions at home. She contributed valuable insights and ideas as to how the robot could be improved. She evaluated the robot based on effectiveness, efficiency and autonomy. Most of the other participants, though, evaluated the robot based on play, creativity and enjoyment.

The participants had strong feelings about what is good for you as you are ageing. The normative aspect of ageing included healthy eating, being active and participating in social activities. Having a robot as an assistive aid evoked internal ambivalence. On the one hand, they could see the benefits of having a robot for others, but on the other, the robot did not correspond to their normative values (the robot implied that one is housebound, lonely, fragile and in need 
of assistance) and their perception of self. As participant 1 stated:

There are two sets of old people: the ones who are interested and invested in the future and the ones who are stuck in the past.

As the excerpt exemplifies, the participants seemed to have a moral judgement on how you should live as an older person, which included keeping up with technological changes. As such, participating in a robot trial at home became a means to do just that.

Sensor and robot logs can appear to be a more objective way to collect data than interviews and observations. Frequent contact with the participants can affect how they talk about and use the robot. However, sensor and robot data does not reveal why and in which situations the participants use the robot and its different functionalities. Through the interviews, observations and the diaries it became clear that the robot was mostly used to show visitors how the robot worked and what it could do. When the participants were at home alone, they did not use the robot, but went back to their everyday routines and habits. Once every day or every few days, they tested the robot since they thought that it was expected of them during the time of the trials.

C. The meaning given to the robot

When people are trying out new technologies they draw on their previous experiences, which may or may not help them [63]. People's expectations also affect how they talk about their experiences and the robot. Our findings indicate that the accountability people ascribe to robots is coloured by their visions of future robots and that they are drawing on their past experiences of other technologies that have become smaller, cheaper and easier to use over time. The participants expressed a belief that robots will be part of future eldercare. Robots were perceived as offering freedom, convenience and the potential to change everyday life for the better. They also expressed fears about robots replacing humans. Concerns for loss of jobs and human care were also raised in previous studies [8]. For the majority of the participants, robots were seen as a supplement to human care and not as a replacement. Participants 1, 2, 4, 5 and 6 all mentioned that the robot they tested represented distraction, mental stimulation and companionship.

I think it's very fun that it talks. It is really beneficial that it talks. This makes it more like a companion; more like a butler. I make the decisions. It [the robot] is undemanding. I can do what I want to do, what suits me and when I want. It [the robot] always has time for me. It becomes undemanding companionship. Many fear that a robot will replace human contact, but a robot does not exclude human contact; instead, the robot will be my servant. If I was bedridden, I would like to have a robot that could help me. It could fetch medicine and read aloud to me. I strongly believe that for those of us who want to take care of ourselves, we would rather have a robot than another person to help us. When you have a robot, you can decide when, what and how (Participant 1).

This extract exemplifies the discrepancy between the attributed abilities and the actual abilities of the socially assistive robot. Attributed abilities refer to the qualities and/or characteristics the participant ascribed the robot. Actual abilities, in contrast, refer to the tangible qualities and characteristics of the robot. The attributed abilities of the socially assistive robot in the extract are characterised by obedience, user control, companionship and that the robot conducts tasks that are currently delegated to humans. The actual abilities of the robot are that it communicates verbally (pre-programed dialogue), and that the user decides when and how long she wants to interact with it. This participant is drawing upon her expectations and preferences for what a robot's capabilities should be when she talks about the robot she is testing. She underlines that the robot is perceived as a companion since it talks and always obeys her. She needs to be in control of the robot and she likes to make the decisions on when and how long she wants to interact with it. The extract also exemplifies the participant's vision that the robot would be able to conduct tasks that are currently delegated to humans-not as a substitute for human contact but as a 24-h supplement that she controls.

The participants' initial expectations of trying out the robot were high. They expected it to be like an artificial companion and butler. The actual abilities of the robot did not meet these high expectations. In spite of this, they still maintained their belief that future robots will be more effective, cheaper and smaller. The participants were drawing on their experience of other technologies such as computers, televisions and mobile phones, all of which have improved and become user-friendlier during their lifetime. Their knowledge of the development and improvements of current technologies, as well as the promise of future robots to support independent living, obscured the actual abilities of the robot when they talked about it.

\section{Discussion}

Aligning our findings with prior work [51,66,67], we have learnt that older people attribute human traits to robots and expect them to behave intelligently and as humans, even though they know that robots are machines. The appearance and behaviour of robots will most likely affect the meaning people ascribe them. In our study the participants wanted the robot to be more social, intelligent and spontaneous, which confirms findings from other home trials $[16,46,70]$. 
These expectations might be managed better by the use of a more machine-like voice and/or a machine-like robot without a head with eyes, as in the case of the robot studied. It is suggested that the behaviour of robots should replicate human-to-human communication in order to ease the human-robot relationship [34]. However, perhaps in line with the "uncanny valley hypothesis regarding aesthetics", robots' behaviours should not resemble humans to the point where the user expects them to behave like humans. Further research is needed on how the design of a robot affects people's perceptions and expectations of a robot. How can different design features and multimodal interactions be used to meet and manage older people's expectations and thereby their response to a robot?

Expectations of technology before usage are likely to originate from mass media or manufacturers, while post-usage of technology is more likely to reveal actual experience [29]. Stafford et al. [62] conducted a study at a retirement village involving a healthcare robot and older people. The participants were invited to use a healthcare robot for a period of 2 weeks. The participants completed a questionnaire before and after the trial. The results showed that the older people had high beliefs and preconceptions of the robot that were altered after they had the experience of using a real robot [62]. In contrast, we observed that our participants held high beliefs in future robots pre-usage, during usage and postusage. Their preconceptions were not significantly altered during the usage phase. A discrepancy was found between how the participants talked about the robot and their actual usages of the robot during the home trials. Initially the robot was used frequently. Over time, however, the participants went back to ordinary routines that did not include the robot. It did not become a part of their everyday life but was used because of the commitment they had made to being a participant.

It is worthwhile to note that the attributed abilities could have been gathered in questionnaires and structured interviews, while the actual usage of the assistive robot would have gone unnoticed. It was by means of observations and diaries that we were able to access the actual usage. The findings show that what the participants said they did differed from what they actually did. The observations and diaries attempted to witness and probe how the participants actually used and integrated the robot in their everyday life. Fundamentally, these qualitative methods provided valuable data on how the participants actually interacted with the robots in practice. There is a risk in relying on questionnaires and structured interviews only: that you will only gather data about the way the participants interpret the human-robot relationship in social, political, historical and economic contexts [14]. The data can also be coloured by the participant's motivation to partake. There can be many reasons as to why our participants praised the robot and the potential of robots in future eldercare: They may have wanted to pleased us (the researchers), or their experience during the home trials may have made them realise the potential of future robots, or they may have wanted to participate in other home trials with robots (something that some of them mentioned).

The actual usage of the robot was affected by the participants' understanding of its functionalities, competing artefacts and its ability to fit into their homes. The participants used the entertainment functions to a higher degree than the physical assistance functions. Most of them were already familiar with touch screens, audio books, the Internet, etc. But they were unfamiliar with the functions for picking up objects from the floor, learning to recognise new objects, and searching for missing or misplaced objects. The participants did not understand the behaviour of the robot when they asked it to execute any of these functions (suitable feedback and visual clues were lacking). However, the functions they were familiar with (Internet, audio book, digital music, radio) did not add any advantages compared to the digital devices they already had. This was the case for all but one of the participants, the one who was not familiar with computers or smart phones. She initially found the robot exciting, but that only lasted a few days.

It is obvious that if robots are meant to be part of the everyday life of older people and tested in their homes, they need to be able to fit into their homes; they need to be able to move around in rooms filled with furniture, carpets, thresholds, and narrow corridors; and they need to be able to handle light reflections from the sun and lamps. If test robots do not fulfil the requirements for object manipulation and navigation, etc., it raises ethical concerns if researchers should invade older people's homes with mobile autonomous robots in pursuit of their research goals. On the other hand, home trials can expose requirements that are not easily exposed in a lab environment.

Studies on robots in natural environments often report on technical malfunctions $[22,41,42,46]$, but not on how they are addressed. We strongly recommend that technical malfunctions are to be addressed and resolved in order to gain a fuller understanding of how people react, change and integrate robots into their everyday life. Not doing so means risking that the robot will not become integrated into everyday life. Laboratory experimental studies are designed to measure attitudes before and after the experiment or the user's behaviour during the experiment, while home trials are designed to understand human-robot interaction in everyday practice [35].

As seen in this empirical case study, the participants' perception of future robots being more capable and better than the existing ones imposes challenges on how to evaluate current robots and how to gather feasible requirements. The relationships between self, others and artefacts (such as robots) are created, recreated and modified during the on- 
going domestication process, which involves mutual change and adaption [38]. The methods used to identify and analyse the potential users' everyday practices with robots need to be dynamic and adaptable. When conducting experimental studies in a laboratory setting, the variables are known. But in an innovation process piloted in potential users' homes, the variables are unknown and the methods need to be able to identify and recognise the unknown. The integration of a robot into everyday practices is affected by other artefacts (e.g. grippers, computers, smart phones in the empirical case study), other humans, routines and daily structures. It is a transformative process in which the user's behaviour and everyday practice change over time in relation to the robot. Home trials are suitable for incremental design processes and rapid prototyping, and provide clues to finding out how a robot should be designed, behave and interact in order for older people to use it and find it useful [12]. Initially, usability studies should be conducted in a lab environment in order to reveal the primary usability and safety issues, as well as the robot's robustness [33].

Home trials are expensive and time consuming to run. Testing a malfunctioning robot in natural environments may not be worth the costs and benefits, if the same insights can be gathered in a lab environment, or if the research study is designed as an experimental study.

Unfortunately, previous research shows that there is a "suppression of relevant, plausible and thus valuable evaluations and assessments regarding the future use of robots by older people" [12]. Under such circumstances older people can become marginalised and the kind of independence and autonomy robots are meant to mediate for older people is constructed and negotiated for them, instead of with them. As a result, the concept of ageing and older people can be negatively formed and transformed. This can lead to the reduction or exclusion of older people from robot use. However, our results show that even though potential older users were involved in the evaluation of a socially assistive robot, they still cultivated the image of other older, weaker and lonelier people than themselves as being in need of robots and held a great belief in the promise of future robots. The imaginings, expectations and visions of future robots affected how the participants talked about the robot but did not portray their actual usage of the robot. To get a holistic picture of the human-robot interaction in natural environments, triangulation of multiple data sources is desired.

Our home trials were an eye-opener and raised important questions related to older people, independent living, and care robots that need to be further considered. Ageing is not a standardised process. We do not all age in exactly the same manner. As we grow older, our experiences of ageing differ. Not only does ageing differ between individuals, but also for the same individual depending on time of day, time of year, situation and context. It also depends on the older individual's experiences of illness or wellness. This raises complex challenges for robots to provide independence, security and empowerment. The experience of using the socially assistive robot provoked internal ambivalence among the participants and raised many ethical issues. One was about the kinds of tasks that are appropriate for socially assistive robots to carry out. Should they be developed to fetch and pick up objects even though it is good for people to move and bend in spite of the difficulties involved? Perhaps it would be better to design robotic functionalities that encourage the user to carry out activities and tasks more safely. Or should the robot be designed to carry out some tasks so that the user could preserve her energy for more meaningful activities? The automation of certain functionalities could in the long-term restrict the autonomy of the older person. On the other hand, encouraging activity could be perceived as paternalistic. Questions about what kind of behaviour is appropriate for robots were also raised among the participants. Should the robot show emotions, and if so, what kind?

\subsection{Limitations}

Studies similar to ours have been criticised for involving a very small number of participants and/or of lacking critical control conditions [10]. However, the home trials were conducted as a part of a design process to collect and interpret potential participants' motivations, needs and wants regarding assistive robots to support independent living. As such, robots need to be viewed as "under construction" and the home trials as a means to understand the local settings including people, other artefacts, everyday routines and structures [48].

Home trials often involve a close relationship between the participants and the research team. This can result in in a socialisation process in which the participants construct their selves. Wenger [69] calls this phenomenon a "community of practice". These communities are groups of individuals who participate in joint activities (such as home trials, especially if they involve frequent visits, technical issues and breakdowns). In so doing, they create a common ground and identity through their engagement in the activity. As a result, the participants may say things they believe the researchers would like to hear, instead of giving their honest opinion. The results show that participating in the home trials became an end in itself and involved increased social communication with others.

All of our participants were women. Unfortunately we were unable to attract any men to participate in the home trials. This might bias and affect our results. Future research should aim to involve both men and women. 


\section{Conclusion}

The field of HRI needs researchers who study robots in the natural environments (people's real lives) for which they are intended. However, these kind of studies are expensive, time consuming and difficult to conduct. The findings from home trials are often less precise than laboratory studies and the findings are dependent on multiple variables such as the design of the robot, the feedback and visual clues provided by the robot to communicate its behaviour, the fit between the robot and the home, the research design, competing artefacts and the participants' motivation to partake. Conclusive findings from a single study are highly unlikely. Together, though, we can build on each other's findings by converging and contrasting our results and thereby increasing our knowledge base. In order to learn from each other we need to present both the successes and failures encountered when carrying out home trials.

Open Access This article is distributed under the terms of the Creative Commons Attribution 4.0 International License (http://creativecomm ons.org/licenses/by/4.0/), which permits unrestricted use, distribution, and reproduction in any medium, provided you give appropriate credit to the original author(s) and the source, provide a link to the Creative Commons license, and indicate if changes were made.

\section{References}

1. Adami I, Antona M, Stephanidis C (2016) Home trials of robotic systems: challenges and considerations for evaluation teams. In: International conference on universal access in human-computer interaction. Springer, pp 291-301

2. Aspers P (2009) Empirical phenomenology: a qualitative research approach (the cologne seminars). Indo Pacific J Phenomenol $9(2): 6-125$

3. Bajones M, Fischinger D, Einramhof P, Wohlkinger W, Papoutsakis K, Mayer P, Panek P, Koertner T, Hofmann S, Argyros A, Pripfl J, Körtner T, Batko-Klein D, Hebesberger D, Weninger M, Gisinger C, Frennert S, Eftring H, Antona M, Adami I, Vincze M (2016) Exploring an assistive social care robot for older adults in realworld homes: lessons learned from field trials with the hobbit robot. Robot Auton Syst (in press)

4. Baxter P, Kennedy J, Senft E, Lemaignan S, Belpaeme T (2016) From characterising three years of HRI to methodology and reporting recommendations. In: The eleventh ACM/IEEE international conference on human robot interation. IEEE Press, pp 391-398

5. Bedaf S, Gelderblom GJ, De Witte L (2015) Overview and categorization of robots supporting independent living of elderly people: what activities do they support and how far have they developed. Assist Technol 27(2):88-100

6. Bemelmans R, Gelderblom GJ, Jonker P, de Witte L (2015) Effectiveness of robot paro in intramural psychogeriatric care: a multicenter quasi-experimental study. J Am Med Dir Assoc 16(11):946-950

7. Broadbent E, Stafford R, MacDonald B (2009) Acceptance of healthcare robots for the older population: review and future directions. Int J Soc Robot 1(4):319-330
8. Broadbent E, Tamagawa R, Patience A, Knock B, Kerse N, Day K, MacDonald BA (2012) Attitudes towards health-care robots in a retirement village. Aust J Ageing 31(2):115-120

9. Broekens J, Heerink M, Rosendal H (2009) Assistive social robots in elderly care: a review. Gerontechnology 8(2):94-103

10. Burton A (2013) Dolphins, dogs, and robot seals for the treatment of neurological disease. Lancet Neurol 12(9):851-852

11. Buxton B (2010) Sketching user experiences: getting the design right and the right design. Morgan Kaufmann, Burlington

12. Compagna D, Kohlbacher F (2015) The limits of participatory technology development: the case of service robots in care facilities for older people. Technol Forecast Soc 93:19-31

13. Crabtree A, Rouncefield M, Tolmie P (2012) Doing design ethnography, Springer, London pp 7-19

14. Creswell JW (2013) Research Design: Qualitative, Quantitative, and Mixed Methods Approaches. Sage publications, Thousand Oaks

15. Dahl TS, Boulos MNK (2013) Robots in health and social care: a complementary technology to home care and telehealthcare? Robotics 3(1):1-21

16. Dautenhahn K, Campbell A, Syrdal DS (2015) Does anyone want to talk to me?: reflections on the use of assistance and companion robots in care homes. In: Proceedings of the 4th international symposium on new frontiers in human-robot interaction. The Society for the Study of Artificial Intelligence and the Simulation of Behaviour (AISB),

17. Davidson K (2002) Gender differences in new partnership choices and constraints for older widows and widowers. Ageing Int 27(4):43-60

18. Dix A (2009) Human-computer interaction. Springer, Berlin

19. Eftring H, Frennert S (2016) Designing a social and assistive robot for seniors. Zeitschrift für Gerontologie und Geriatrie, 49(4):274281

20. Fasola J, Matarić MJ (2012) Using socially assistive human-robot interaction to motivate physical exercise for older adults. Proc IEEE 100(8):2512-2526

21. Fazekas G, Tóth A, Rumeau P, Zsiga K, Pilissy T, Dupourque V (2012) Cognitive-care robot for elderly assistance: preliminary results of tests with users in their homes. In: AAL Forum, Netherlands

22. Fernaeus Y, Håkansson M, Jacobsson M, Ljungblad S (2010) How do you play with a robotic toy animal?: a long-term study of pleo. In: Proceedings of the 9th international conference on interaction design and children. ACM, pp 39-48

23. Fischinger D, Einramhof P, Wohlkinger W, Papoutsakis K, Mayer P, Panek P, Koertner T, Hofmann S, Argyros A, Vincze M (2013) Hobbit-the mutual care robot. In: Workshop on assistance and service robotics in a human environment workshop in conjunction with IEEE/RSJ international conference on intelligent robots and systems

24. Forlizzi J, DiSalvo C (2006) Service robots in the domestic environment: a study of the roomba vacuum in the home. In: Proceedings of the 1st ACM SIGCHI/SIGART conference on human-robot interaction. ACM, pp 258-265

25. Frennert S (2014) Older people and the adoption of innovations: a study of the expectations on the use of social assistive robots and telehealthcare systems, Licentiate Thesis Lund: department of design sciences, Lund University. Available http://lup.lub.lu.se/ search/record/5051136

26. Frennert S, Östlund B (2014) Review: seven matters of concern of social robots and older people. Int J Soc Robot 6(2):299-310

27. Graneheim UH, Lundman B (2004) Qualitative content analysis in nursing research: concepts, procedures and measures to achieve trustworthiness. Nurse Educ Today 24(2):105-112 
28. Heerink M, Kröse B, Evers V, Wielinga B (2010) Assessing acceptance of assistive social agent technology by older adults: the almere model. Int J Soc Robot 2(4):361-375

29. Hong S, Thong JY, Tam KY (2006) Understanding continued information technology usage behavior: a comparison of three models in the context of mobile internet. Decis Support Syst 42(3):18191834

30. Jøranson N, Pedersen I, Rokstad AMM, Ihlebæk C (2015) Effects on symptoms of agitation and depression in persons with dementia participating in robot-assisted activity: a cluster-randomized controlled trial. J Am Med Dir Assoc 16(10):867-873

31. Kidd CD, Taggart W, Turkle S (2006) A sociable robot to encourage social interaction among the elderly. In: Robotics and automation, 2006. ICRA 2006. Proceedings 2006 IEEE international conference on. IEEE, pp 3972-3976

32. Kidd CD, Breazeal C (2008) Robots at home: understanding longterm human-robot interaction. In: Intelligent robots and systems, 2008. IROS 2008. IEEE/RSJ international conference on. IEEE, pp 3230-3235

33. Kjeldskov J, Stage J (2004) New techniques for usability evaluation of mobile systems. Int J Hum Comput Stud 60(5):599-620

34. Krämer NC, von der Pütten A, Eimler S (2012) Human-agent and human-robot interaction theory: similarities to and differences from human-human interaction. In: Zacarias M, de Oliveria J.V. (eds) Human-computer interaction: the agency perspective. Springer, pp 215-240

35. Kuutti K, Bannon LJ (2014) The turn to practice in HCI: Towards a research agenda. In: Proceedings of the 32 nd annual ACM conference on Human factors in computing systems. ACM, pp 3543-3552

36. Lammer L, Huber A, Weiss A, Vincze M (2014) Mutual care: how older adults react when they should help their care robot. In: AISB2014: Proceedings of the 3rd international symposium on new frontiers in human-robot interaction

37. Lesnoff-Caravaglia G (2007) Gerontechnology: growing old in a technological society. Charles C Thomas Publisher Limited, Srpingfield

38. Lie M, Sørensen KH (1996) Making technology our own?: domesticating technology into everyday life. Scandinavian University Press, Oslo

39. Mayer P, Panek P (2013) A social assistive robot in an intelligent environment. BioMed Tech 58:1

40. Mayer P, Panek P (2014) Towards a multi-modal user interface for an affordable Assistive Robot. In: International conference on universal access in human-computer interaction. Springer, pp 680 691

41. Michalowski MP, DiSalvo C, Busquets D, Hiatt LM, Melchior NA, Simmons R, Sabanovic S (2006) Socially distributed perception. In: Proceedings of the 1st ACM SIGCHI/SIGART conference on human-robot interaction. ACM, pp 349-350

42. Mutlu B, Forlizzi J (2008) Robots in organizations: the role of workflow, social, and environmental factors in human-robot interaction. In: Human-robot interaction (HRI), 2008 3rd ACM/IEEE international conference on. IEEE, pp 287-294

43. Newell AF, Arnott J, Carmichael A, Morgan M (2007) Methodologies for involving older adults in the design process. In: Stephanides $\mathrm{S}$ (ed) Universal access in human computer interaction. coping with diversity 4th international conference on universal access in human-computer interaction, UAHCI 2007, Held as Part of HCI international 2007, Beijing, China, July 22-27, 2007, Proceedings, Part I pp. 982-989

44. Neven L (2010) 'But obviously not for me': robots, laboratories and the defiant identity of elder test users. Sociol Health Illn 32(2):335347

45. Norman DA (2013) The design of everyday things: revised and expanded edition. Basic books, New York
46. Orejana JR, MacDonald BA, Ahn HS, Peri K, Broadbent E (2015) Healthcare robots in homes of rural older adults. In: International conference on social robotics. Springer, pp 512-521

47. Panek P, Mayer P (2015) Challenges in adopting speech control for assistive robots. In: Wichert R, Klausing $\mathrm{H}$ (eds) Ambient assisted living advanced technologies and societal change. Springer, pp 3 14. doi:10.1007/978-3-319-11866-6_1

48. Pols J (2012) Care at a distance. Amsterdam University Press, Amsterdam

49. Pripfl J, Körtner T, Batko-Klein D, Hebesberger D, Weninger M, Gisinger C (2016) Social service robots to support independent living. Zeitschrift für Gerontologie und Geriatrie, 49(4), 282-287

50. Pripfl J, Körtner T, Batko-Klein D, Hebesberger D, Weninger M, Gisinger C, Frennert S, Eftring H, Antona M, Adami I (2016) Results of a real world trial with a mobile social service robot for older adults. In: The eleventh ACM/IEEE international conference on human robot interation. IEEE Press, pp 497-498

51. Reeves B, Nass C (1996) How people treat computers, television, and new media like real people and places. CSLI Publications, Stanford

52. Rehrl T, Troncy R, Bley A, Ihsen S, Scheibl K, Schneider W, Glende S, Goetze S, Kessler J, Hintermueller C, Wallhoff F (2012) The ambient adaptable living assistant is meeting its users. In: AAL Forum 2012

53. Robinson H, MacDonald B, Kerse N, Broadbent E (2013) The psychosocial effects of a companion robot: a randomized controlled trial. J Am Med Dir Assoc 14(9):661-667

54. Rodeschini G (2011) Gerotechnology: a new kind of care for aging? An analysis of the relationship between older people and technology. Nurs Health Sci 13(4):521-528

55. Rogers EM (1995) Diffusion of innovations. Free Press, New York

56. Rogers Y (2012) HCI theory: classical, modern, and contemporary. Synthesis lectures on human-centered informatics, vol 5. Morgan \& Claypool publishers, London, pp 1-129

57. Šabanović S, Reeder S, Kechavarzi B (2014) Designing robots in the wild: In situ prototype evaluation for a break management robot. J Hum Robot Interact 3(1):70-88

58. Schroeter C, Mueller S, Volkhardt M, Einhorn E, Huijnen C, van den Heuvel H, van Berlo A, Bley A, Gross H-M (2013) Realization and user evaluation of a companion robot for people with mild cognitive impairments. In: Robotics and automation (ICRA), 2013 IEEE international conference on. IEEE, pp 1153-1159

59. Sharkey A, Sharkey N (2012) Granny and the robots: ethical issues in robot care for the elderly. Ethics Inf Technol 14(1):27-40

60. Silverstone R, Hirsh E (2003) Consuming technologies-media and information in domestic spaces. Routledge, London

61. Sparrow R, Sparrow L (2006) In the hands of machines? The future of aged care. Minds Mach 16(2):141-161

62. Stafford RQ, MacDonald BA, Jayawardena C, Wegner DM, Broadbent E (2014) Does the robot have a mind? Mind perception and attitudes towards robots predict use of an eldercare robot. Int J Soc Robot 6(1):17-32

63. Suchman L (2007) Human-machine reconfigurations: plans and situated actions. Cambridge University Press, Cambridge

64. Sung J, Christensen HI, Grinter RE (2009) Robots in the wild: understanding long-term use. In: Human-robot interaction (HRI), 2009 4th ACM/IEEE international conference on. IEEE, pp 45-52

65. Sung J, Grinter RE, Christensen HI (2009) Pimp my roomba: designing for personalization. In: Proceedings of the SIGCHI conference on human factors in computing systems. ACM, pp 193-196

66. Taggart W, Turkle S, Kidd CD (2005) An interactive robot in a nursing home: preliminary remarks. In: Towards social mechanisms of android science: a COGSCI workshop 2005

67. Turkle S, Breazeal C, Dasté O, Scassellati B (2006) First Encounters with Kismet and Cog: children respond to relational artifacts". 
In: Digital media: transformations in human communication, Messaris P, Humphreys L (eds) Peter Lang Publishing, New York, 1-20

68. Vallor S (2011) Carebots and caregivers: sustaining the ethical ideal of care in the twenty-first century. Philos Technol 24(3):251-268

69. Wenger E (1999) Communities of practice: learning, meaning, and identity. Cambridge University Press, Cambridge

70. Wu Y-H, Cristancho-Lacroix V, Fassert C, Faucounau V, de Rotrou J, Rigaud A-S (2016) The attitudes and perceptions of older adults with mild cognitive impairment toward an assistive robot. J Appl Gerontol 35(1):3-17

71. Wu YH, Fassert C, Rigaud AS (2011) Designing robots for the elderly: appearance issue and beyond. Arch Gerontol Geriatr 54(1):121-126

72. Young JE, Hawkins R, Sharlin E, Igarashi T (2009) Toward acceptable domestic robots: applying insights from social psychology. Int J Soc Robot 1(1):95-108

Susanne Frennert B.Sc in Cognitive Science, M.Sc in Human Factors and Ergonomics and Ph.D. in Rehabilitation Engineering. Her research interests are ambient assistive living and social robots in relation to older people in ordinary homes and at care homes.
Håkan Eftring Ph.D., Assistant Professor in Rehabilitation Engineering at the Department of Design Sciences at Lund University, Sweden. His previous education is a Master of Science in Mechanical Engineering and a Licentiate degree in Manufacturing Systems. His research interests are social and assistive robotics for people with physical disabilities and old people, universal design and user-centered and situated design and evaluation methods.

Britt Östlund is a Professor at the Royal Institute of Technology, KTH, and leading Technology in Healthcare research in collaboration with the Red Cross University College. For thirty years she has been dedicated to research and development in aging, technology and design, was responsible for ten years for the Ageing and design program at Lund University, where she still holds a visiting professorship. 